\title{
Ultrasound biomicroscopic analysis of pseudophakic pupillary block glaucoma induced by Soemmering's ring
}

\author{
Hiroshi Kobayashi, Mio Hirose, Kaori Kobayashi
}

\begin{abstract}
Aim-To perform ultrasound biomicroscopic analysis of pseudophakic pupillary block glaucoma induced by lens capsule and a Soemmering's ring and its resolution, and to elucidate the pathophysiology of this glaucoma.

Methods-A woman with pseudophakic pupillary block glaucoma underwent successful neodymium:YAG (Nd:YAG) laser photodisruption of the lens capsule through a laser iridotomised coloboma with resolution of the pupillary block. The Humphrey ultrasonic biomicroscope model 840 was employed to observe the anterior segment before and after laser photodisruption.

Results-Ultrasound biomicroscopic examination revealed the intraocular lens (IOL) was displaced forward, shallowing the central anterior chamber. The anterior hyaloid face was relatively posterior to the posterior capsule. The iris was in apposition to the anterior capsule, which was in touch with the IOL optics. A massive Soemmering's ring, which extended from the IOL optics to the ciliary processes, was displaced anteriorly. The Soemmering's ring consisted of several tightly packed layers. The ciliary processes rotated anteriorly. After Nd:YAG laser photodisruption of the capsule, ultrasound biomicroscopic images showed the resolution of the pupillary block and the anterior rotation of the ciliary process. The Soemmering's ring moved posteriorly, and the layers in the ring became loose.

Conclusions-A massive Soemmering's ring may one of potential causes of pupillary block after cataract surgery. Ultrasound biomicroscopy is potentially useful as a non-invasive diagnostic technique for clinical diagnosis and differentiation between Soemmering's ring induced pupillary block glaucoma and other forms of pupillary block.
\end{abstract}

(Br F Ophthalmol 2000;84:1142-1146)

Pupillary block is the most common cause of angle closure following cataract surgery and intraocular lens (IOL) implantation. ${ }^{12}$ The pupil can become occluded by any surface that lies behind or in front of the iris. The causes of pupillary block after cataract surgery include leaky wound, postoperative iridocyclitis, dense, impermeable anterior hyaloid membrane (malignant glaucoma), adhesion between the pupil and IOL (pupillary block glaucoma), adhesion among the capsule, IOL and iris (posterior pupillary block glaucoma), pupillary block by air or silicone, inadequate iris openings, swollen lens material behind the iris, choroidal detachment and haemorrhage, scleral collapse, free vitreous block, and anterior chamber haemorrhage. . $^{3-7}$

After cataract surgery and IOL implantation, in the peripheral part of the capsular bag, where the remaining anterior capsule rests on the posterior capsule, proliferation of lens epithelium results in a thick structure known as a Soemmering's ring. It sometimes develops into having the appearance of a string of sausages. ${ }^{8} 9$ However, no Soemmering's ring has been reported to cause pupillary block.

Conventional methods such as gonioscopy and ultrasonic biometry present difficulties in evaluating dimensions of the anterior chamber, ${ }^{10-12}$ and their use can lead to an incorrect diagnosis. ${ }^{13}$ High resolution ultrasound biomicroscopy provides precise measurements and produces images that cannot be obtained by conventional methods. ${ }^{10-12}{ }^{14-21}$

The following patient who underwent cataract surgery and posterior chamber IOL implantation was initially seen with elevated intraocular pressure, an extremely shallow anterior chamber, peripheral iridocorneal apposition, and anterior movement of IOL. Findings from ultrasound biomicroscopy and subsequent neodymium:YAG (Nd:YAG) laser treatment indicated that a Soemmering's ring and lens capsule induced pupillary block with aqueous accumulation behind the posterior capsule and collapse of the posterior chamber. This is the first time that a Soemmering's ring has been demonstrated as a possible cause of pseudophakic pupillary block.

\section{Patient and methods}

ULTRASOUND BIOMICROSCOPIC MEASUREMENTS An ultrasonic biomicroscope model 840 (ZeissHumphrey, San Leandro, CA, USA) with a 50 $\mathrm{Hz}$ transducer was employed. An eye cup was used to separate the eyelids after a drop of $0.5 \%$ proxymetacaine (proparacaine) had been applied. The eye cup was filled with $2.5 \%$ methylcellulose as a coupling medium. Scanning was performed in eight directions, placing the probe close to the area of interest. We repeated each measurement five times to confirm reproducibility and eliminate artefacts.

Anterior chamber depth was measured as an axial distance from the internal corneal surface to the lens surface. Ultrasound biomicroscopic 

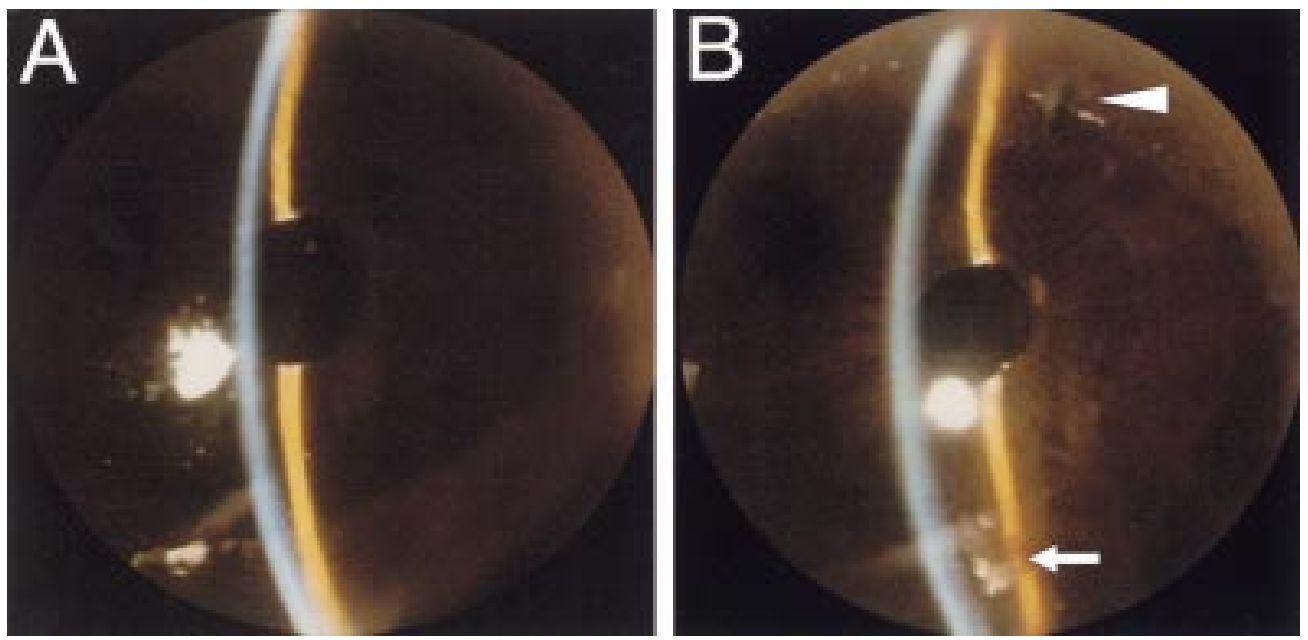

Figure 1 Slit lamp photographs of anterior segment. (A) Before neodymium:YAG laser photodisruption of the capsule. The anterior chamber is centrally shallow with peripheral iridocorneal touch. (B) The anterior chamber is deep after the treatment. A small white secondary cataract mass (arrow) was observed to move out of a retroirideal space into the anterior chamber through the iridotomised hole (arrowhead).

cross sectional views through the angle region were obtained for most of the measurements, which were made at 12:00, 1:30, 3:00, 4:30, 6:00, 7:30, 9:00, and 10:30 o'clock positions; views were as vertical as possible, as determined from the screen image. The following variables were measured: anterior chamber depth, trabecular iris angle, angle opening distance at 250 and $500 \mu \mathrm{m}$ from the scleral spur (AOD250 and AOD500), iris thickness, trabecular-ciliary distance, iris-ciliary process distance, iris-zonule distance, scleral-iris angle, and scleral-ciliary process angle, as described by Pavlin et al. ${ }^{10-12}$ Before Nd:YAG laser photodisruption of the capsule and Soemmering's ring, the mean and standard deviation was calculated from the values at all measured positions. After the treat-
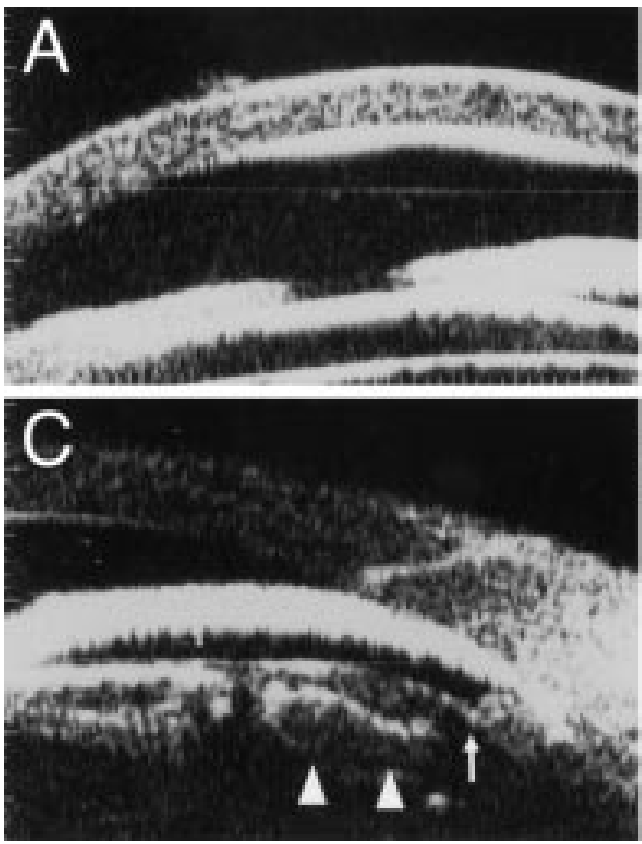

ment, the mean and standard deviation was calculated from the values at all measured positions except the 4:30 position, where the anterior synechia was found.

STATISTICAL ANALYSIS

Values are expressed as the mean (SD) and as a range. Unless otherwise specified, data were analysed by paired, unilateral $t$ tests. A level of $\mathrm{p}$ $<0.05$ was accepted as statistically significant.

\section{Results}

CASE REPORT

A 82 year old Japanese woman presented with a visual loss in the right eye in association with marked visual field defect and high intraocular pressure. She underwent uncomplicated cata-

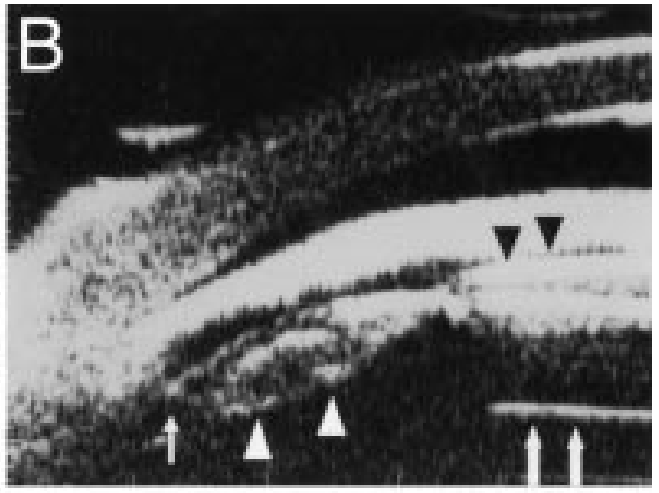

Figure 2 Ultrasound biomicroscopic images before laser treatment. (A) The central anterior chamber is shallow. The iris is in apposition to the anterior capsule, which was in touch with the IOL. (B, C) Peripheral iridocorneal apposition is present. The ciliary body (small white arrow) is rotated anteriorly. A massive Soemmering's ring (white arrowheads), which extends from the edge of the IOL otics to the ciliary processes, displaced anteriorly. Several layers in the ring are tightly packed. The iris (black arrowheads) is in touch with the IOL optics. The anterior hyaloid face (white arrows) is relatively posterior to the posterior capsule diaphragm. 
ract phacoemulsification and intraocular lens (MZ60BD, Alcon, Fort Worth, TX, USA) implantation of both eyes 3 years ago, and Nd:YAG (Nd:YAG) laser posterior capsulotomy of the right eye a year ago.

The best corrected visual acuity was 0.05 right eye and 1.0 left eye. The Humphrey visual field test of the right eye showed a visual field defect of upper half, including the fixation. The intraocular pressure was $48 \mathrm{~mm}$ $\mathrm{Hg}$ right eye and $16 \mathrm{~mm} \mathrm{Hg}$ left eye. Slit lamp biomicroscopy revealed that the anterior chamber was shallow centrally with peripheral iridocorneal apposition (Fig 1A). Several pits were found in the IOL corresponding to Nd:YAG laser capsulotomy. The posterior capsule was fibrotic with a central defect by $\mathrm{Nd}$ :YAG laser posterior capsulotomy. Indentation gonioscopy showed the angle to be closed $360^{\circ}$. B scan ultrasonography of the posterior segment was unremarkable.

Ultrasound biomicroscopy was employed to obtain cross sectional images of the anterior segment of the right eye. Pretreatment images showed peripheral iridocorneal touch with appositional angle closure (Fig 2B and C). The central view revealed that the IOL was displaced forward, shallowing the central anterior chamber (Fig 2A). The anterior hyaloid face was relatively posterior to the posterior capsule, and a distance between the IOL and the anterior hyaloid face was $613 \mu \mathrm{m}$. The iris was in apposition to the anterior capsule, which was in touch with the IOL optics. An ellipse whose posterior surface was covered by the posterior capsule was found from the edge of the optics to the ciliary process (Fig 2B and C). The ellipse was considered as a Soemmering's ring. In the Soemmering's ring, there were tightly packed several horizontal layers, which appeared to consist of fine fibrils. The entire Soemmering's ring moved anteriorly. No adhesion was found between the Sommering's ring and the iris. The ciliary processes rotated anteriorly (Fig 2B and C). Results of examination of the ciliochoroidal space did not show the presence of supraciliary or choroidal effusions.

Laser iridotomy for the right eye was performed at the 12:00 position. The positions of the iris, IOL, and ciliary body were unchanged. Through a coloboma by laser iridotomy, Nd:YAG laser photodisruption of the capsule and Soemmering's ring was performed. The anterior chamber immediately deepened with resolution of the iridocorneal touch (Fig 1B). A small white mass was observed to move out of the retroirideal space into the anterior chamber through the iridotomised hole. The intraocular pressure reduced to $10 \mathrm{~mm} \mathrm{Hg}$. Gonioscopic examination showed the angle to be open with peripheral anterior synechia at the $4: 30$ position.

Ultrasound biomicroscopic examination showed that, immediately after laser surgery, the central anterior chamber depth deepened to $2796 \mu \mathrm{m}$ and most of the angle opened (Fig 3A). Peripheral anterior synechia was seen at
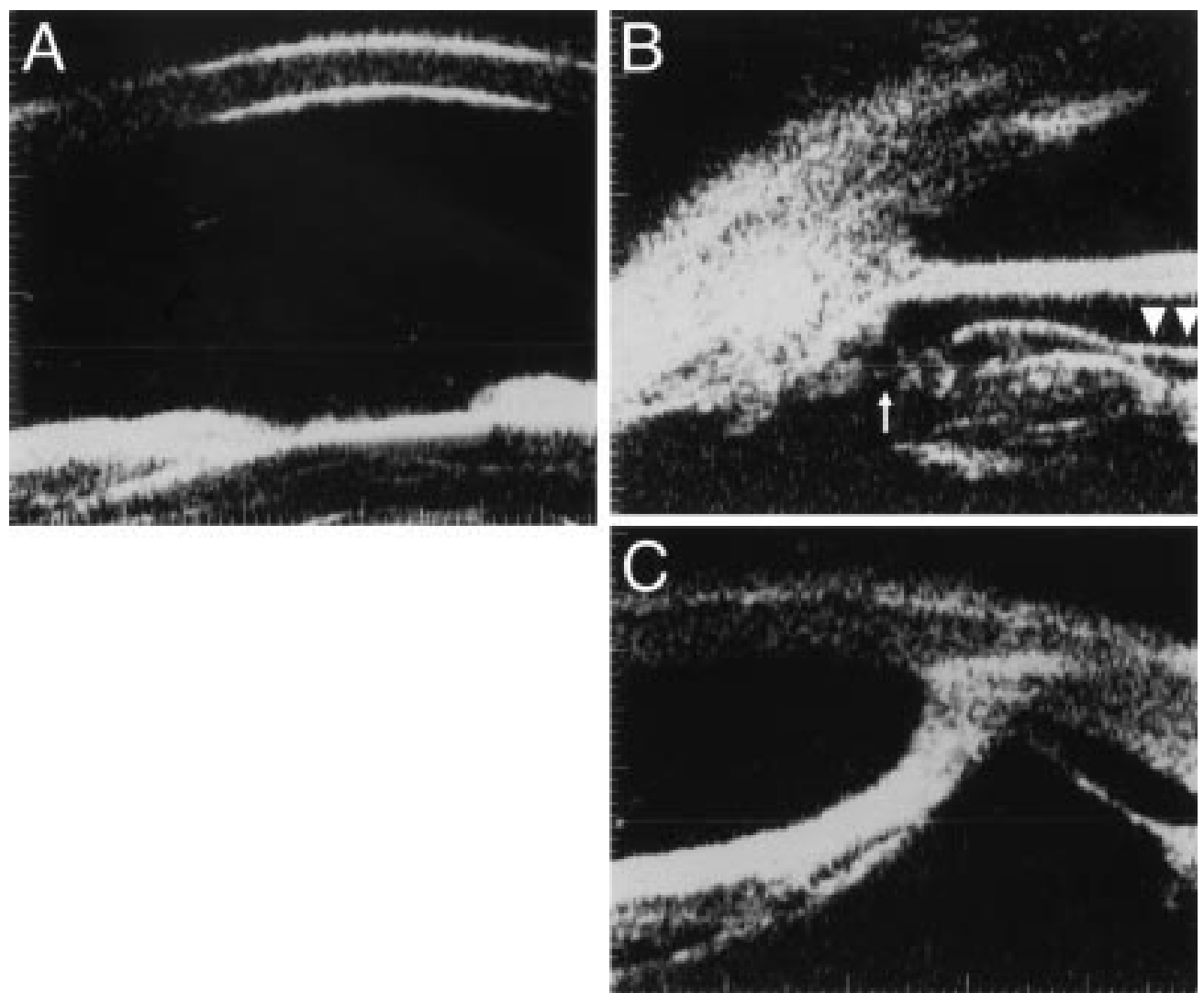

Figure 3 Immediately after laser surgery. (A) The central anterior chamber is deep. (B) The angle is open. Ultrasound biomicroscopic examination showed the resolution of the touch between the iris, anterior capsule (arrowheads) and IOL optics, and the anterior rotation of the ciliary process (small arrows). The whole Soemmering's ring and IOL optics moved posteiorly. Several layers in the ring which were tightly packed became loose. (C) Peripheral anterior synechia at the 4:30 position. 
Table 1 Dimensions (SD) of anterior segment before and after resolution

\begin{tabular}{llll}
\hline & Before resolution & After resolution & p Value \\
\hline Anterior chamber depth $(\mathrm{mm})$ & $1192(25)$ & $2796(25)$ & $<0.0001$ \\
$\quad$ Range & 1169 to 1219 & 2769 to 2819 & \\
Trabecular-iris angle (degree) & $0.84(1.47)$ & $43.30(7.38)$ & $<0.0001$ \\
$\quad$ Range & 0 to 3.44 & 32.11 to 52.34 & \\
AOD250 $(\mu \mathrm{m})$ & $2(6)$ & $253(51)$ & $<0.0001$ \\
$\quad$ Range & 0 to 13 & 188 to 312 & \\
AOD500 $(\mu \mathrm{m})$ & $13(13)$ & $429(31)$ & $<0.0001$ \\
$\quad$ Range & 0 to 31 & 381 to 463 & \\
Trabecular-ciliary process distance $(\mu \mathrm{m})$ & $567(195)$ & $1430(160)$ & $<0.0001$ \\
Range & 431 to 902 & 1144 to 1506 & \\
Iris-ciliary process distance $(\mu \mathrm{m})$ & $157(53)$ & $506(156)$ & $<0.0001$ \\
$\quad$ Range & 94 to 203 & 381 to 762 & \\
Iris-zonule distance $(\mu \mathrm{m})$ & $200(33)$ & $577(109)$ & $<0.0001$ \\
$\quad$ Range & 175 to 256 & 488 to 758 & \\
Scleral-iris angle (degree) & $8.3(10.89)$ & $30.43(4.49)$ & 0.0002 \\
$\quad$ Range & 0.82 to 23.8 & 23.5 to 35.82 & \\
Scleral-ciliary process angle (degree) & $4.05(9.40)$ & $40.30(12.02)$ & $<0.0001$ \\
$\quad$ Range & -8.19 to 13.71 & 27.18 to 55.04 & \\
\hline
\end{tabular}

the $4: 30$ position and the capsule was adherent to the iris (Fig 3C). The IOL optics and the whole Sommering's ring moved posteriorly. In the Sommering's ring, the layers which were tightly packed before the laser treatment became loose (Fig 3B). Ultrasound biomicroscopic images showed the resolution of the touch between the iris, anterior capsule, and IOL optics, and the anterior rotation of the ciliary processes.

Table 1 showed dimensions of anterior segment before and after the Nd:YAG laser photodisruption of the capsule and Soemmering's ring. Anterior chamber depth increased from $1192 \mu \mathrm{m}$ to $2796 \mu \mathrm{m}$, trabecular-iris angle from $0.84^{\circ}$ to $43.3^{\circ}, \mathrm{AOD} 250$ from $2 \mu \mathrm{m}$ to $253 \mu \mathrm{m}$, AOD 500 from $13 \mu \mathrm{m}$ to $429 \mu \mathrm{m}$, trabecular-ciliary process distance from 567 $\mu \mathrm{m}$ to $1430 \mu \mathrm{m}$, iris-ciliary process distance from $157 \mu \mathrm{m}$ to $506 \mu \mathrm{m}$, iris-zonule distance from $200 \mu \mathrm{m}$ to $577 \mu \mathrm{m}$, scleral-iris angle from $8.3^{\circ}$ to $30.43^{\circ}$, and scleral-ciliary process angle from $4.05^{\circ}$ to $40.3^{\circ}$; a significant difference was found in all measured dimensions between before and after the treatment. For 12 months after the laser treatment, the intraocular pressure of the right eye was controlled at 18 $\mathrm{mm} \mathrm{Hg}$ or lower.

\section{Discussion}

We demonstrated ultrasound images of pseudophakic pupillary block glaucoma induced by a Soemmering's ring and lens capsule, and its resolution.

The ultrasound biomicroscopy showed an oval structure which spread out from the edge of IOL optics to the ciliary processes and which was covered by the posterior capsule. The oval structure could not be observed directly because we were unable to dilate the pupil; however, it was considered as a Soemmering's ring, because a white mass moving into the anterior chamber after laser capsulotomy appeared part of secondary cataract. The Soemmering's ring, posterior capsule covering the IOL optics, and IOL optics might prevent aqueous humour from escaping from the posterior chamber into the anterior chamber. Before laser treatment, accumulation of aqueous humour behind the Soemmering's ring and posterior capsule caused the entire Soemmering's ring and IOL optics to move forward and several layers in the ring to become packed tightly. Nd:YAG laser photodisruption of the lens capsule allowed aqueous humour to pass from the posterior chamber into the anterior chamber, leading to the resolution of the pupillary block.

The differential diagnosis includes malignant glaucoma (posterior aqueous misdirection glaucoma), anterior pupillary block glaucoma (pupillary block), posterior pupillary block glaucoma (anterior aqueous misdirection glaucoma), choroidal haemorrhage, and ciliochoroidal effusion with anterior rotation of the ciliary body. ${ }^{7}$ In pupillary block, iris bombé is typically present and the IOL is in a relatively normal position. ${ }^{17}{ }^{19} \mathrm{~A}$ formed posterior chamber and no anterior rotation of the ciliary body are found. After laser iridotomy, the iris contour flattens with minimal change in IOL position. In this patient, the posterior segment was normal. The configuration of the anterior segment in our patient was entirely different from that in pseudophakic pupillary block, and laser iridotomy did not relieve the pupillary block.

Clinical diagnosis and differentiation among Sommering's ring induced pupillary block, posterior pupillary block glaucoma, and malignant glaucoma are difficult. ${ }^{5-7}$ In all, the pathophysiology of aqueous misdirection lies in the altered relation and dysfunctional aqueous dynamics occurring behind the iris plane. 5-7 17 22-24 $^{20}$ This results in aqueous humour misdirection that eventually accumulates in the vitreous or retrocapsular space. This causes anterior rotation of the ciliary processes and shallowing of the anterior chamber. In malignant glaucoma, the volume and pressure within vitreous increases significantly; the anterior hyaloid membrane moves forward. In the posterior pupillary block glaucoma, adhesion among the iris, IOL, and capsule prevents aqueous humour from passing into the anterior chamber. In this patient, the anterior hyaloid becomes displaced backwards and the canal of Petit becomes an exaggerated space. Ultrasound microscopy showed a massive Soemmering's ring extending to the ciliary processes and no adhesion between the iris and capsule, which was found in the posterior pupillary block glaucoma. Nd:YAG laser capsulotomy through the iridotomised coloboma relieved the pupillary block, whereas laser iridotomy was not effective. Therefore, the massive, broad Soemmering's ring and capsule might hinder escape of aqueous humour into the anterior chamber.

Tomey et al reported that Nd:YAG laser posterior capsulotomy is an effective alternative to laser iridotomy for managing selected pupillary block in pseudophakic eyes. ${ }^{55}$ With an IOL in place, capsulotomy is reported to be effective outside of the lens optic or through one of the positioning holes. ${ }^{525}$ Capsulotomy behind the lens may be ineffective, since the lens itself may prevent the aqueous humour from gaining access from the retrocapsular space to the anterior chamber. This patient already underwent Nd:YAG laser capsulotomy, and laser iridotomy did not relieve 
the pupillary block. Capsulotomy was performed through the iridotomised hole, because inability of dilating the pupil prevented us from observing capsule outside of the lens optic or the positioning holes. Immediately after photodisruption of the capsule and Soemmering's ring through the iridotomised hole, the anterior chamber deepened and the iridocorneal touch resolved with part of a Soemmering's ring moving into the anterior chamber through the iridotomised hole.

In the patient with pseudophakic pupillary block glaucoma induced by a Soemmering's ring, ultrasound biomicroscopy provided cross sectional images of the iris, anterior and posterior capsules, IOL, Soemmering's ring and ciliary body, and their relative positions before and after laser treatment. Ultrasound biomicroscopy provides images which show the pathophysiology of pupillary block glaucoma induced by a Soemmering's ring and is potentially useful as a non-invasive diagnostic technique for clinical diagnosis and differentiation among a Soemmering's ring induced pupillary block glaucoma and other forms of pseudophakic pupillary block glaucoma.

1 Francois J. Aphakic glaucoma. Ann Ophthalmol 1974;6:429. 2 Layden WE. Glaucoma and intraocular lens implantation. In: Ritch $\mathrm{R}$, Shields MB, eds. The secondary glaucomas. St In: Ritch R, Shields

3 Jaffe NS, Jaffe MS, Jaffe GF. Cataract surgery and its complications. 5th ed. St Louis: Mosby, 1990:370.

4 Verhoeff $\mathrm{FH}$. The nature and origin of the pigmented streaks caused by separation of the choroid. $7 A M A$ 1931;97:1873-7.

5 Tomey KF, Traverso CE. Neodymium-YAG posterior capsulotomy for the treatment of aphakic and pseudophakic pupillary block. Am f Ophthalmol 1987;104:502-7.

6 Tomey KF, Traverso CE, Antonios SR, et al. Mechanisms of pupillary block. (Reply to Dr RJ Mackool.) Arch Ophthalmol 1988;106:167.

7 Tomey KF, Traverso CE. The glaucomas in aphakia and pseudophakia. Surv Ophthalmol 1991;36:79-112.
8 McDonnell PJ, Zarbin MA, Green WR. Posterior capsule opacification in pseudophakic eyes. Ophthalmology 1983; 90:1548-53.

9 McDonnell PJ, Stark WJ, Green WR. Posterior capsule opacification: a specular microscopic study. Ophthalmology 1984;91:853-6.

10 Pavlin CJ, Harasiewicz K, Foster FS. Ultrasound biomicroscopy of anterior segment structures in normal and glaucomatous eves. Am 7 Ophthalmol 1992;113:381-9.

11 Pavlin CJ, McWhae JA, McGowan HD, et al. Ultrasound biomicroscopy of anterior segment tumors. Ophthalmology 1992;99:1220-8.

12 Pavlin CJ, Harasiewicz K, Sherar MD, et al. Clinical use of ultrasound biomicroscopy. Ophthalmology 1991;98:28795.

13 Makabe R. Vergleichende Untersuchungen der Kammerwinkelweite mit Echographie und Gonioskopie. Klin Monatsbl Augenheilkd 1989;194:6-9.

14 Tello C, Liebmann J, Potash SD, et al. Measurement of ultrasound biomicroscopy images: intraobserver and interobserver reliability. Invest Ophthalmol Vis Sci 1994;35:54952.

15 Pavlin CJ, Sherar MD, Foster FS. Subsurface ultrasound microscopic imaging of the intact eye. Ophthalmology 1990;97:244-50.

16 Pavlin CJ, Ritch R, Foster FS. Ultrasound biomicroscopy in plateau iris syndrome. Am f Ophthalmol 1992;113:390-5.

17 Tello C, Chi T, Shepps G, et al. Ultrasound biomicroscopy in pseudophakic malignant glaucoma. Ophthalmology 1993;100:1330-4.

18 Potash SD, Tello C, Liebmann J, et al. Ultrasound biomicroscopy in the pigment dispersion syndrome. Ophthalmology 1994;101:332-9.

19 Aslanides IM, Libre PE, Silverman RH, et al. High frequency ultrasound imaging in pupillary block glaucoma. Br f Ophthalmol 1995;79:972-6.

20 Kobayashi H, Kobayashi K, Kiryu J, et al. Ultrasound biomicroscopic analysis of the effect of pilocarpine on the anterior chamber angle. Graefes Arch Chin Exp Ophthatmol 1997;235:425-30.

21 Kobayashi H, Kobayashi K, Kiryu J, et al. Ultrasound biomicroscopic measurements of anterior chamber angle in premature infants. Br F Ophthalmol 1997;81:460-4.

22 Lynch MG, Brown RH, Michels RG, et al. Surgical vitrectomy for pseudophakic malignant glaucoma. Am $\mathcal{F}$ Ophthalmol 1986;102:149-53.

23 Ruben S, Tsai J, Hitchings RA. Malignant glaucoma and its management. Br f Ophthalmol 1997;81:163-7.

24 Trope GE, Palvin CJ, Bau A, et al. Malignant glaucoma: clinical and ultrasound biomicroscopic features. Ophthalmology 1994;101:1130-5.

25 Risco JM, Tomey KF, Perkins TW. Laser capsulotomy through IOL positioning holes in anterior aqueous misdirection. Arch Ophthalmol 1989;107:1569-72. 